\title{
Shells of revolution under arbitrary loading and the use of fictitious densities in dynamic relaxation
}

A. C. CASSELL

\section{Dr Winifred L. Wood, Department of Mathematics, University of Reading}

It would be interesting if the Author could say more about the estimation of $a$, the minimum eigenvalue of the stiffness matrix, which is necessary for the evaluation of the optimum value of the damping coefficient $K$. The governing differential equations for the shell do not appear to be such that there is a uniform variation in density with changes in mesh size. Were there two separate runs with zero damping for the coarse and finer mesh or was the result from one used to estimate the other and if so by what formula?

55. Lynch et al. ${ }^{13}$ could not observe any simple periodic form in the run with zero damping in the system they were investigating. To obtain a good estimation of the minimum eigenvalue they had to resort to Fourier Analysis which seems uneconomic. They also experimented by iterating to get the dominant eigenvalue of the shift matrix:

$$
\mathbf{A}-q I
$$

where $\mathbf{A}$ is the (positive definite) stiffness matrix. The dominant eigenvalue will be:

$$
-(q-a) \text { if } q>\frac{1}{2}(a+b) \quad \text {. . . . . . . }
$$

56. If the eigenvalue of $\mathbf{A}$ next in magnitude to $a$ is $a+\epsilon$, then the speed of convergence will depend on the smallness of:

$$
\frac{q-a-\epsilon}{q-a} \text { or of } \frac{b-q}{q-a} \cdot . \quad . \quad . \quad . \quad .
$$

The best strategy is to make these equal i.e.:

$$
q=\frac{1}{2}(a+b+\epsilon)
$$

but as we do not know $a$ or $\epsilon$ we have to estimate. Lynch et al. ${ }^{13}$ found experimentally for their system that the optimum $q$ was $55 \%$ of $b$ (which they also found by iteration). Has the Author met any problems where $\omega_{0}$ was difficult to observe from the run with zero damping and has he tried any other methods?

57. With the Author's method of fictitious densities the stiffness matrix has its coefficients adjusted so that each row has the same numerical sum of coefficients. If the matrix is further adjusted by dividing these sums so that each row has a numerical sum of coefficients equal to unity then the Gershgörin upper bound for the spectral radius is unity also. It has been shown ${ }^{14}$ that (provided $K$ can be estimated successfully) DR has a faster asymptotic rate of convergence than the Chebyshev: semiiterative method with fixed parameter which it closely resembles.

\section{Dr K. R. Rushton, University of Birmingham}

The Author makes an important statement at the end of $\S 38$ which confirms my experience in dealing with a number of problems in elasticity and fluid mechanics and I have found that the physical picture of the method is a great help in devising methods of speeding the convergence.

Paper published: Proc. Instn civ. Engrs, 1970, 45 (January) 65-78. 
59. For example, in the non-linear problems of the large deflexion of plates the need for fictitious densities can be seen from a physical argument. In this problem both in-plane and out-of-plane displacements occur with two second order equations describing the in-plane equilibrium, and one fourth order equation for the out-ofplane equilibrium. ${ }^{15}$ For these two equations there are different stability criteria; with the second order equations the permissible time increment is proportional to the mesh interval and the square root of the density, whilst the fourth order equation has a permissible time increment proportional to the square of the mesh interval and the square root of the density. See equation (34). To ensure that the same maximum time step applies to each equation fictitious densities must be introduced in the in-plane directions.

60. Fictitious densities were also used in a study of the stress concentration factors in grooved plates. ${ }^{16}$ Due to the presence of high stresses a graded mesh was used with small mesh intervals in the neighbourhood of the groove. Since the stability criterion given by equation (34) depends on the mesh intervals in the $x$ and $y$ directions, then the density at each node must be chosen so that $\Delta t$ is constant over the whole plate. This approach proved to be successful, but the changed densities did lead to an increase in the period of the oscillation resulting in an increase in the number of iterations required. For example, in the grooved plate problem when the region has a graded mesh with the smaller mesh interval equalling a fifth of the largest, then the number of iterations is roughly twice that for the same region with a uniform mesh having a spacing equal to the largest of the graded mesh.

61. If I had been aware of the stability equations described in the Appendix it might have been possible to overcome difficulties which have occurred in certain problems. For example, in the large deflexion of plates with no in-plane boundary support, ${ }^{17}$ instability problems arose which could only be overcome by reducing the time increment. However, by calculating the densities in a manner similar to equation (32) it seems probable that this difficulty could be overcome. Difficulties also arose with solutions to the Laplace equation with complex boundary conditions ${ }^{18}$ and the modification of the densities to allow for the form of the boundary conditions may well be the key to solving this problem without reducing the time increment.

62. In general I have found that the dynamic relaxation technique is an excellent method of solving a wide variety of field problems. It is particularly valuable for non-linear equations and also for complex boundary conditions. ${ }^{\mathbf{1 9}}$

\section{Mr J. H. Davidson, W. S. Atkins and Partners}

Did Dr Cassell consider using large deflexion theory which would introduce nonlinear terms such as $\frac{1}{2}(\partial w / \partial x)^{2}$ into equation (1) and $T_{x}\left(\partial^{2} w / \partial x^{2}\right)$ into equation (15)? From Fig. 5 it appears that the maximum value of $\partial w / \partial x$, just below the knuckle, is about $5 \times 10^{-3}$. This would result in an unrelaxed stress of about $400 \mathrm{lb} / \mathrm{sq}$. in., which is over $10 \%$ of the unrelaxed temperature stresses.

64. For structures whose dynamic behaviour approximates to that of a damped spring-mass system, I have calculated the response to a step loading for various values of damping factor, and hence the time that elapses before subsequent deflexions all lie within a specified range on either side of the static solution. The results are summarized in Table 1 . The $0.5 \%$ error criterion leads to:

$$
N=6 \cdot 6 / K \quad(\text { See } \$ 17) .
$$

65. Most structures have a more complex dynamic response, and more iterations are required. However, $I$ feel that an iteration time of 1.5 natural periods is unwarranted for most engineering problems, where the mathematical models do not simulate the actual behaviour with $100 \%$ accuracy.

66. I have previously used the initial disturbance method to estimate the time increment for non-standard cases. 
Table 1. Optimum damping and calculation time for damped spring-mass systems

\begin{tabular}{|c|c|c|c|c|}
\hline Acceptable error & . . & $2 \%$ & $1 \%$ & $0.5 \%$ \\
\hline Fraction of critical damping & . & $0 \cdot 8$ & 0.85 & 0.9 \\
\hline Fraction of free period & . & 0.4 & $0 \cdot 5$ & 0.6 \\
\hline
\end{tabular}

Table 2. Comparison of criteria for time increment

\begin{tabular}{|c|c|c|c|c|c|}
\hline Case & & $\begin{array}{l}\text { Gershgörin } \\
\text { bound }\end{array}$ & $\begin{array}{c}\text { Initial d } \\
\left(\Delta T_{\text {orit }}\right)_{1}\end{array}$ & $\begin{array}{l}\text { urbance } \\
\left(\Delta T_{\text {crit }}\right)_{2}\end{array}$ & Coefficients \\
\hline $\begin{array}{l}\text { Beam bending } . \\
\text { Plate bending } \\
\text { Rod axial stress . } \\
\text { Plate membrane stress } \\
\text { Body stress . } \\
\text { Cylinder dilatation : } \\
\text { Sphere dilatation }\end{array}$ & 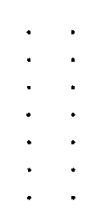 & $\begin{array}{l}1 \cdot 732 \\
0 \cdot 866 \\
1.000 \\
0 \cdot 707 \\
0.577 \\
1 \cdot 414 \\
1 \cdot 000\end{array}$ & $\begin{array}{l}2 \cdot 000 \\
1.096 \\
1.000 \\
0 \cdot 816 \\
0 \cdot 707 \\
1.414 \\
1.000\end{array}$ & $\begin{array}{l}1.758 \\
1.032 \\
1.000 \\
0.849 \\
0.756 \\
1.414 \\
1.000\end{array}$ & $\begin{array}{l}\Delta x^{2} / c h \\
\Delta x^{2} / c h \\
\Delta x / c \\
\Delta x / c \\
\Delta x / c \\
R / c \\
R / c\end{array}$ \\
\hline
\end{tabular}

Notes: by dilatation is meant uniform radial oscillation without bending.

For simplicity $\Delta z=\Delta y=\Delta x$ and $\nu=0$ hence $c^{2}=E / \rho$.

The values are for the damping factor $(K / 2)$ to be zero, generally $(K / 2)<0 \cdot 1$.

67. At time $t=0$, let all stresses, deflexions and velocities be zero, except at node $j$ where velocity $\left(\dot{u}_{j}\right)_{0}=v$ say. By using the finite difference equations with zero damping, the velocity $\left(\dot{u}_{1}\right)_{1}$ at time $t=\Delta t$ can be calculated. For marginal stability let $\left(\dot{u}_{1}\right)_{1}=-v$. Hence, $\Delta t$ can be found.

68. For the beam bending example, the finite difference equation is:

$$
\left(\dot{w}_{\mathrm{j}}\right)_{1}=\left(\dot{w}_{\mathrm{j}}\right)_{0}-\frac{E I \Delta t}{A \rho \Delta x^{4}}\left\{\begin{array}{c}
w_{\mathrm{j}-2}-4 w_{\mathrm{j}-1}+6 w_{\mathrm{j}} \\
-4 w_{\mathrm{j}+1}+w_{\mathrm{j}+2}
\end{array}\right\}_{0}
$$

and deflexions at $t=\Delta t / 2$ are all zero except for $\left(w_{1}\right)_{0}=v \cdot \Delta t$. For critical $\Delta t$ :

$$
-v=v-\frac{6 E I \Delta t^{2}}{A \rho \Delta x^{4}} v
$$

Hence $\left(\Delta t_{\text {cr } 1 \mathrm{t}}\right)_{1}=\Delta x^{2} / \sqrt{ }(3)(A \rho / E I)^{1 / 2}$, or, writing $E / \rho=c^{2}$, and with $E I=b h^{3} / 12$, $A=b h$ :

$$
\left(\Delta t_{\mathrm{crit}}\right)_{1}=\frac{2 \Delta x^{2}}{c h}
$$

69. If a similar process is carried out for the condition that $\left(\dot{w}_{1}\right)_{2}=+v$, it is found that:

$$
\left(\Delta t_{\mathrm{crit}}\right)_{2}=\frac{1 \cdot 758 \Delta x^{2}}{c h}
$$

70. Values obtained by this method are compared in Table 2 with those obtained by the Gershgörin bound, which for direct stress waves are identical with those obtained by Forsythe and Wasow for dilatation waves. (Section 26 of 'Finite Difference Methods for Partial Differential Equations'.)

71. It seems that the smallest initial disturbance value may be an upper bound for $\Delta t$, and the Gershgörin bound value a lower bound. This may be borne 


\section{DISCUSSION}

out by Dr Cassell's experience described in $\S 39$. However, my experience is that, for curved structures, the various direct and bending stress waves interact, and a lower value of $\Delta t$ must be used.

72. For plate membrane stress, the Forsythe and Wasow criterion is obtained for the equation:

$$
\ddot{u}=c^{2}\left(\frac{\partial^{2} u}{\partial x^{2}}+\frac{\partial^{2} u}{\partial y^{2}}\right)
$$

whereas DR is:

$$
\ddot{u}=c^{2}\left(\frac{\partial^{2} u}{\partial x^{2}}+\frac{1}{2} \frac{\partial^{2} u}{\partial y^{2}}+\frac{1}{2} \frac{\partial^{2} v}{\partial x \partial y}\right)
$$

For a dilatation wave, the two are identical since then $\partial v / \partial x=\partial u / \partial y$. Generally, there will be waves of both dilatation and distortion, and the latter move at about half the speed of the former.

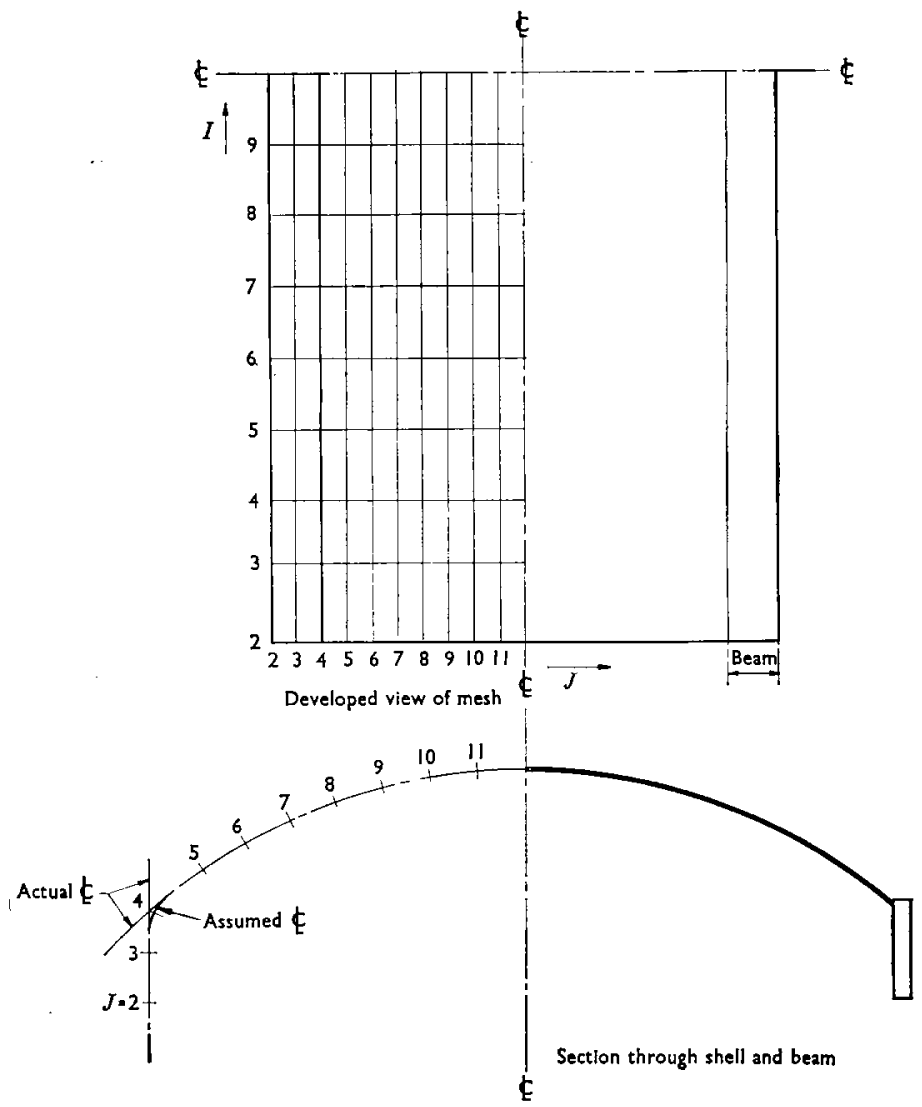

Fig. 7. Mesh for cylindrical shell with edge beams 
Table 3. Stiffnesses for thin reinforced concrete sections

\begin{tabular}{|c|c|c|c|c|c|}
\hline & \multicolumn{3}{|c|}{ Membrane forces } & \multicolumn{2}{|c|}{ Moments } \\
\hline & \multicolumn{2}{|c|}{ Direct } & \multirow[t]{2}{*}{ Shear } & \multirow[t]{2}{*}{ Direct } & \multirow[t]{2}{*}{ Torsion } \\
\hline & Tension & $\begin{array}{c}\text { Com- } \\
\text { pression }\end{array}$ & & & \\
\hline $\begin{array}{l}\text { Normal stiffness } \\
\text { Factor for reinforced concrete }\end{array}$ & $\begin{array}{c}E h \\
1 \cdot 5 r\end{array}$ & $\begin{array}{c}E h \\
1+1 \cdot 5 r\end{array}$ & $\begin{array}{l}E h / 2 \\
1.5 r\end{array}$ & $\begin{array}{c}E h^{3} / 12 \\
2 \cdot 5 r\end{array}$ & $E h^{3} / 24$ \\
\hline
\end{tabular}

Note: $r=m p$, where $m$ is modular ratio, and $p$ is percentage of reinforcement, same each layer top and bottom mat.

73. The DYSH program incorporates two uncommon features. First, large deflexion theory, which, combined with the vibrations of dynamic relaxation, gives values for shell buckling loads in better accordance with test results; and second, a bilinear stress-strain law for reinforced concrete which gives a truer representation of working load stresses.

74. The basic equations are those for shallow thin curved and twisted plates (i.e. mesh geometry assumed to be that of a developable surface). The two special features can be incorporated optionally. The finite differences used are all first order, the variables falling naturally on to a rectangular interlacing mesh similar to that of Fig. 2.

75. With regard to boundary conditions and edge beams each of the three deffexions and one rotation at each edge of the shell can be completely free or completely fixed. Deep edge beams can be represented by thickened strips of shell, using the standard shell equations. The angular discontinuity of some $60^{\circ}$ between shell and edge beam is replaced by a circular arc of length one mesh interval as shown in Fig. 7. This procedure was amply justified by the results of an analysis of a case for which Gibson $^{20}$ gives an exact solution.

76. The behaviour of reinforced concrete is represented approximately by applying factors to the uncracked plain concrete stiffnesses as shown in Table 3. The stiffness for direct stress depends upon the sign of the stress, and is recalculated for $T_{x}$ and $T_{y}$ at each node at each iteration. To avoid oscillation, alterations are made gradually, tending asymptotically to the true value.

77. $\Delta t$ is calculated by the program, using the plate membrane stress criterion (Gershgörin bound of Table 3). Fictitious densities can be used for the $\dot{w}$ normal velocities, so as to make either the radial dilatation or the plate bending criterion critical as well. It has been found necessary to reduce $\Delta t$ if fictitious densities are used, a reduction of $30 \%$ (to overcome interaction between the three criteria); if large deflexion terms are used, a reduction of $15 \%$; and if concrete stress-strain relationships are used, a reduction of $12 \%$. Damping factor $K=8 / N$, which for optimum $N$ usually gives virtually dead-beat settlement.

78. Fig. 8 shows diagrammatically the load-deflexion paths obtained by analytical methods, using either infinitesimal or large deflexion theories. By contrast, test results show buckling occurring at about 0.2 of the Euler load $\boldsymbol{P}_{\mathrm{E}}$.

79. The DYSH program was used to calculate the response of a perfectly elastic thin cylinder (with $\nu=0$ ), under various axial loadings, to very small random radial loads, using large deflexion equations and zero damping. It was found that the radial oscillations were stable for $P<0.25 P_{E}$, and unstable for $P>0.275 P_{E}$. There are good reasons for believing that the instability corresponded to buckling.

80. The program was used in the design of a multishell roof, consisting of seven cylindrical shells with partially prestressed edge beams, spanning between 


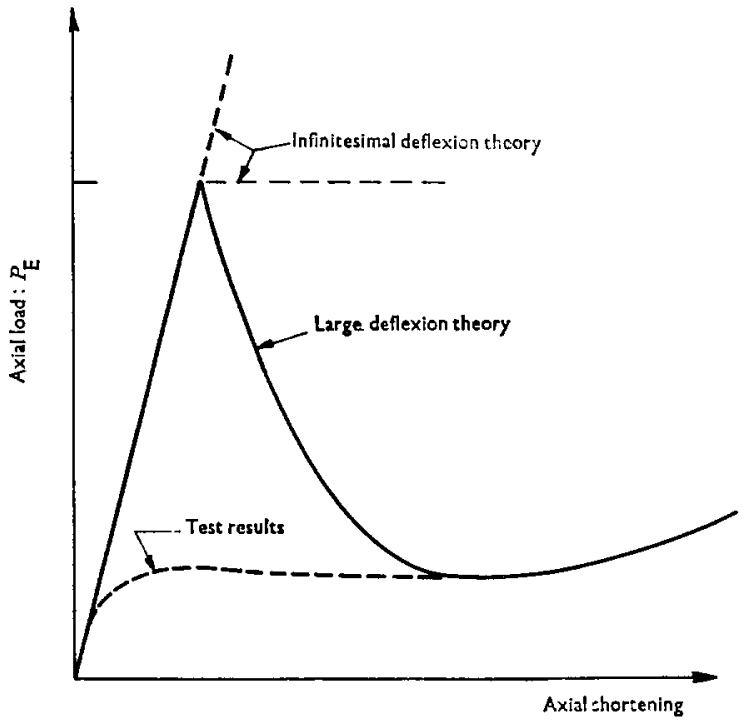

Fig. 8. Local buckling of axially loaded cylinder

gables. End and interior shells were analysed. It was found that when the reinforced concrete section stiffnesses were used, the tension of the edge-beam soffit was markedly reduced, so also were shear stresses and bending moments generally, as compared with the case of constant elastic modulus.

81. More significantly, it was found that the factor of safety against buckling was halved because of the great reduction of bending stiffness of a lightly reinforced concrete section after cracking. Accordingly, the shells were designed with considerably more bending reinforcement than was needed for the working load moments.

82. When the non-linear large deflexion theory and concrete stress-strain relationships are used, the final solution may depend upon the loading history. If it is wished to simulate slowly applied proportional loading, it may be sufficient to use a higher than critical damping factor. This aspect requires further investigation.

\section{Dr A. C. Cassell}

Dr Wood with her helpful discussion has, in effect, pointed out that whilst it is possible to control the numerical stability by an explicit formula, the second parameter of iteration, $K$, is not so defined. This is not surprising because a reasonably close bound to the minimum eigenvalue of a sparse matrix cannot be found with the same ease as the Gershgörin bound for the maximum eigenvalue. Dr Wood is correct, the change in fictitious densities did not vary uniformly with changes in mesh size. The arithmetic mean of all the fictitious densities was used to calculate $\omega_{0}$ and hence $K$ for the fine mesh, and this empirical approach worked satisfactorily.

84. Lynch et al. have used a different form of DR in which the densities were proportional to the main diagonal terms of the finite element stiffness matrix, which gave a different dynamic response. No difficulty has been encountered in the estimation of $\omega_{0}$ from a computer run with low (not zero) damping.

85. Dr Rushton's contribution is of considerable value in showing non-linear applications of DR. If fictitious densities are used to study the large deflexion 
behaviour of plates, then these densities would need to be recalculated as the coefficients in the equations change during the computation. However, fictitious densities would probably be of help in these problems.

86. The large deflexion terms were not included in the shell equations because preliminary calculations showed that the terms would not significantly affect the displacements. It is agreed that large deflexion behaviour must always be considered, and the description by Mr Davidson of shell buckling analysis by DR is welcomed for its novelty.

87. I agree with the late Professor Otter, who recommended 1.5 natural periods for the length of iteration on the ground that the saving in cost of labour in resubmitting a program, which had not properly settled down, exceeded any cost of computer time wasted. Shorter times can be used for subsequent computer runs.

88. The initial disturbance method is of value in being an alternative systematic method for calculating the time increment. Except where $a \nless \hbar b$ the Gershgörin method gives a lower bound to the time increment and thus numerical stability.

\section{References}

14. WoOD WINIFRed L. Note on dynamic relaxation (to appear in Int. J. Num. Meth. Eng.).

15. Rushton K. R. Large deflexion of variable thickness plates. Int. J. mech. Sci., 1968, 70, 723-735.

16. Rushton K. R. The dynamic relaxation method used for stress analysis. Recent advances in stress analysis. Conference of Royal Aero. Soc., 1968.

17. Rushton K. R. Dynamic relaxation solution for the large deflexion of plates with specified boundary stresses. Inl strain Analysis, 1969, 4, 75-80.

18. Rushton K. R. and Laing L. M. Oscillatory lift interference in low-speed rectangular tunnels with porous-slotted roof and floor. Aeronautical Research Council 31 615, Nov., 1969.

19. Rushton K. R. Simply supported plates with corners free to lift. Jnl strain Analysis, 1969, 4, 306-311.

20. Gibson J. E. Design of shell roofs. Spon, London. 1968. 